\title{
CLASS GROUPS, TOTALLY POSITIVE UNITS, AND SQUARES
}

\author{
H. M. EDGAR, R. A. MOLLIN ${ }^{1}$ AND B. L. PETERSON
}

\begin{abstract}
Given a totally real algebraic number field $K$, we investigate when totally positive units, $U_{K}^{+}$, are squares, $U_{K}^{2}$. In particular, we prove that the rank of $U_{K}^{+} / U_{K}^{2}$ is bounded above by the minimum of (1) the 2-rank of the narrow class group of $K$ and (2) the rank of $U_{L}^{+} / U_{L}^{2}$ as $L$ ranges over all (finite) totally real extension fields of $K$. Several applications are also provided.
\end{abstract}

1. Notation and preliminaries. Let $K$ be an algebraic number field and let $C_{K}$ denote the ideal class group in the ordinary or "wide" sense. Let $C_{K}^{(+)}$denote the "narrow" ideal class group of $K$. Thus $\left|C_{K}\right|=h_{K}$, the "wide" class number of $K$, and $\left|C_{K}^{(+)}\right|=h_{K}^{(+)}$, the "narrow" class number of $K$. We denote the Hilbert class field of $K$ by $K^{(1)}$; i.e., $\operatorname{Gal}\left(K^{(1)} / K\right) \cong C_{K}$, and we denote the "narrow" Hilbert class field by $K^{(+)}$; i.e., $\operatorname{Gal}\left(K^{(+)} / K\right) \cong C_{K}^{(+)}$. Moreover we adopt the "bar" convention to mean "modulo squares"; for example, $\bar{C}_{K}=C_{K} / C_{K}^{2}$.

Let $U_{K}$ denote the group of units of the ring of algebraic integers of $K$. When $K$ is totally real, we let $U_{K}^{+}$denote the subgroup of totally positive units; i.e., those units $u$ such that $u^{\sigma}>0$ for all embeddings $\sigma$ of $K$ into $\mathbf{R}$. Finally, for any finite abelian group $A$ with $\mid \overline{A \mid}=2^{d}, d$ is called the 2-rank of $A$, which we denote by $\operatorname{dim}_{2} A$.

2. Results. We are concerned with the question:

(*) When is $U_{K}^{+}=U_{K}^{2}$ ?

We begin by observing that $\operatorname{dim}_{2}\left(\bar{U}_{K}^{+}\right)=0$ if and only if $K^{(+)}=K^{(1)}[6$, Theorem 3.1, p. 203]. In particular, when $K$ is a real finite Galois extension of 2-power degree over $Q$, then $\operatorname{dim}_{2}\left(\bar{U}_{K}^{+}\right)=0$ if and only if $N\left(U_{K}\right)=\{ \pm 1\}[3$, Theorem 1, p. 166]. For example, when $K$ is a real quadratic field, then $\operatorname{dim}_{2}\left(\bar{U}_{K}^{+}\right)=0$ if and only if the norm of the fundamental unit is -1 . Necessary and sufficient conditions (in terms of the arithmetic of the underlying quadratic field $K$ ) for the existence of a fundamental unit of norm -1 are unknown (see [8]). This indicates the difficulty of solving (*) for the simplest even degree case. In this regard one may ask whether $(*)$ is equivalent to such a norm statement for other fields. In a recent letter to the authors, V. Ennola answered (*) for cyclic cubic fields $K$ as follows: Let $\varepsilon$ be a norm positive unit of $K$ such that -1 and the conjugates of $\varepsilon$ generate the unit group. Then $\operatorname{dim}_{2}\left(\bar{U}_{K}^{+}\right)=0$ if and only if $\varepsilon$ is not totally positive. However, as with

Received by the editors February 18, 1985, and, in revised form, September 20, 1985.

1980 Mathematics Subject Classification (1985 Revision). Primary 11R80, 11R27, 11R29; Secondary 11R37, 11R32.

${ }^{1}$ This author's research is supported by N.S.E.R.C. Canada. 
the quadratic field case, the latter does not readily translate into arithmetic conditions on the underlying cyclic cubic field $K$. We have not been able to verify that such a norm condition holds for a larger class of fields. For example, it would be interesting to investigate this question for quartic fields. However, we do have the following result which gives an upper bound on $\bar{U}_{K}^{+}$in terms of $\bar{U}_{L}^{+}$for a totally real extension field $L$ of $K$. For example, this will allow us to translate the norm criterion from a quadratic field to any of its totally real number field extensions. We note that the following generalizes [3, Theorem 2, p. 168].

TheOREM 2.1. Suppose $Q \subseteq K \subseteq L$ with $L$ totally real and finite over $Q$. Then $\operatorname{dim}_{2} \bar{U}_{K}^{+} \leqslant \operatorname{dim}_{2} \bar{U}_{L}^{+}$.

Proof. First we show that $K^{(1)} \subseteq L^{(1)}$ and $K^{(+)} \subseteq L^{(+)}$. Both $K^{(1)} L / L$ and $K^{(+)} L / L$ are Galois extensions with abelian Galois groups, since $\operatorname{Gal}\left(K^{(1)} L / L\right) \cong$ $\operatorname{Gal}\left(K^{(1)} /\left(K^{(1)} \cap L\right)\right)$, which is a subgroup of the class group $C_{K}=\operatorname{Gal}\left(K^{(1)} / K\right)$, and $\operatorname{Gal}\left(K^{(+)} L / L\right) \cong \operatorname{Gal}\left(K^{(+)} / K^{(+)} \cap L\right)$, which is a subgroup of the narrow class group $C_{K}^{(+)}=\operatorname{Gal}\left(K^{(+)} / K\right)$. By [1] we have that all $L$-primes are unramified in $K^{(1)} L$ and all finite $L$-primes are unramified in $K^{(+)} L$. It follows that $K^{(1)} \subseteq$ $K^{(1)} L \subseteq L^{(1)}$ and $K^{(+)} \subseteq K^{(+)} L \subseteq L^{(+)}$.

We show next that $K^{(+)} \cap L^{(1)}=K^{(1)}$. The inclusion $K^{(1)} \subseteq K^{(+)} \cap L^{(1)}$ is clear. Since $K \subseteq K^{(+)} \cap L^{(1)} \subseteq K^{(+)}$, we see that $K^{(+)} \cap L^{(1)}$ is an abelian extension of $K$ and that every finite $K$-prime is unramified in $K^{(+)} \cap L^{(1)}$. Moreover, since $L$ is totally real, then $L^{(1)}$ is totally real, and since $K^{(+)} \cap L^{(1)} \subseteq L^{(1)}$, then all infinite $K^{(+)} \cap L^{(1)}$-primes are real. Hence $K^{(+)} \cap L^{(1)} \subseteq K^{(1)}$, and we have shown that $K^{(+)} \cap L^{(1)}=K^{(1)}$.

The following diagram illustrates our situation.

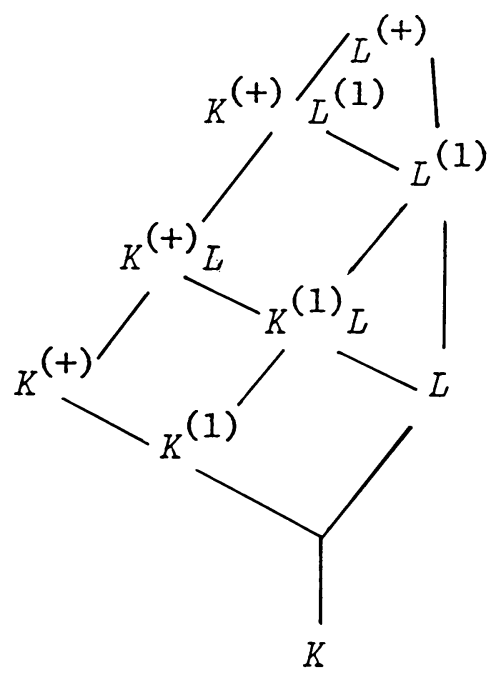

Since $K^{(+)} \cap L^{(1)}=K^{(1)}$ and $K^{(+)} / K^{(1)}$ is Galois, the extensions $K^{(+)} / K^{(1)}$ and $L^{(1)} / K^{(1)}$ are linearly disjoint, and $\left|K^{(+)} L^{(1)}: L^{(1)}\right|=\left|K^{(+)}: K^{(1)}\right|$. Thus $2^{\operatorname{dim}_{2} \bar{U}_{K}^{+}}=$ $\left|K^{(+)}: K^{(1)}\right|=\left|K^{(+)} L^{(1)}: L^{(1)}\right|$ divides $\left|L^{(+)}: L^{(1)}\right|=2^{\operatorname{dim}_{2} \bar{U}_{L}^{+}}$, and so $\operatorname{dim}_{2} \bar{U}_{K}^{+} \leqslant$ $\operatorname{dim}_{2} \bar{U}_{L}^{+}$. Q.E.D. 
With $L$ and $K$ as in Theorem 2.1 we have the following

Corollary 2.2. Let $p$ be a prime and let $K$ be a subfield of $L=Q\left(\zeta_{p}+\zeta_{p}^{-1}\right)$. If the class number of $Q\left(\zeta_{p}\right)$ is odd, then $\operatorname{dim}_{2} \bar{U}_{K}^{+}=\operatorname{dim}_{2} \bar{U}_{L}^{+}=0$.

Proof. A classical result of Kummer (for example see [8, p. 128]) is that $\operatorname{dim}_{2} \bar{U}_{L}^{+}=0$ whenever the class number of $Q\left(\zeta_{p}\right)$ is odd. Therefore the result now follows from Theorem 2.1. Q.E.D.

It is worth noting at this juncture that as a result of recent work of Shimura [10] Kummer's classical result cited in the proof of Corollary 2.2 is now extended to $\operatorname{dim}_{2}\left(\bar{U}_{L}^{+}\right)=0$ if and only if the class number of $Q\left(\zeta_{p}\right)$ is odd. Here $L=Q\left(\zeta_{p}+\zeta_{p}^{-1}\right)$ as in Corollary 2.2.

Furthermore, by [3, p. 175], if $L=Q\left(\zeta_{f}+\zeta_{f}^{-1}\right)$, where $f$ is composite, then $\operatorname{dim}_{2} \bar{U}_{L}^{+}>0$, and $N\left(U_{L}\right)=\{1\}$.

An application of Theorem 2.1 is the following

EXAMPLE 2.3. Let $K=Q(\alpha)$ where the minimal polynomial of $\alpha$ over $Q$ is $x^{3}-x^{2}-234 x+729$. Then $K$ has conductor 703 ; i.e., the minimal cyclotomic field containing $K$ is $Q\left(\zeta_{703}\right)$, so $K \subseteq L=Q\left(\zeta_{703}+\zeta_{703}^{-1}\right)$. It can be shown that $\operatorname{dim}_{2} \bar{U}_{K}^{+}=2$. Thus by Theorem $2.1 \operatorname{dim}_{2} \bar{U}_{L}^{+} \geqslant 2$. For a list of such examples of cyclic cubic fields the reader may consult [2].

Next we prove

Proposition 2.4. Let $K$ be a totally real algebraic number field. Then $\operatorname{dim}_{2} \bar{U}_{K}^{+} \leqslant$ $\operatorname{dim}_{2} C_{K}^{(+)}$.

Proof. From [6, Theorem 3.1, p. 203] we have that

$$
\operatorname{dim}_{2}\left(\bar{U}_{K}^{+}\right)=\operatorname{dim}_{2} \operatorname{Gal}\left(K^{(+)} / K^{(1)}\right) \leqslant \operatorname{dim}_{2} \operatorname{Gal}\left(K^{(+)} / K\right)=\operatorname{dim}_{2}\left(C_{K}^{+}\right) \text {. Q.E.D. }
$$

We note that, in general, it is not correct to claim that $\operatorname{dim}_{2} \bar{U}_{K}^{+} \leqslant \operatorname{dim}_{2} C_{K}$. For example, if $K=Q(\sqrt{3})$, then $\operatorname{dim}_{2} C_{K}=0$. However, $K(\sqrt{-1})$ is unramified except at the real primes of $K$, and in fact $K^{(+)}=K(\sqrt{-1})$ [6, Theorem 3.10, p. 210]. Thus $\operatorname{dim}_{2}\left(\bar{U}_{K}^{+}\right)=\operatorname{dim}_{2} C_{K}^{(+)}=1$. However, under more restrictive hypotheses it is possible to achieve $\operatorname{dim}_{2} \bar{U}_{K}^{+} \leqslant \operatorname{dim}_{2} C_{K}$.

TheOREM 2.5 (ORIAT [9]). Let $K$ be a finite real Galois extension of $Q$ with Galois group of odd exponent $n$ and suppose that -1 is congruent to a power of 2 modulo $n$. Then $\operatorname{dim}_{2} \bar{U}_{K}^{+} \leqslant \operatorname{dim}_{2} C_{K}$. Moreover $\operatorname{dim}_{2} C_{K}^{(+)}=\operatorname{dim}_{2} C_{K}$.

In particular, when $h_{K}$ is odd, we have $\operatorname{dim}_{2} \bar{U}_{K}^{+}=0$. (See [5] for an independent proof of this fact, distinct from that of [9]. The authors of [5] were unaware of the existence of [9] at the time of that writing.)

In the presence of Proposition 2.4, $\operatorname{dim}_{2} \bar{U}_{K}^{+} \leqslant \operatorname{dim}_{2} C_{K}$ is of course an immediate consequence of $\operatorname{dim}_{2} C_{K}=\operatorname{dim}_{2} C_{K}^{(+)}$.

We now exhibit a proof of Theorem 2.5 in the case where $K$ is abelian. This is a simple proof based on the self-duality results established in [11]. Before so doing we need to set the stage with some additional notation and concepts. 
Let $O_{K}$ denote the ring of integers of an algebraic number field $K$. We define $\alpha \in O_{K}$ to be singular if the ideal $(\alpha)$ is the square of an ideal. Moreover $\alpha$ is called odd if $(\alpha)$ is relatively prime to (2). Furthermore, $\alpha$ is called primary if it is odd and $\alpha \equiv \zeta^{2}(\bmod 4)$ for some $\zeta \in O_{K}$. (For details and further development on these concepts we refer the reader to Hecke [4, pp. 217-237].) We denote by $O_{K}^{0}$ the singular primary numbers and note that by [4, Theorem 120, p. 137] we have $\mu \in O_{K}^{0}$ with $\mu \notin O_{K}^{2}$ if and only if $K \subsetneq K(\sqrt{\mu}) \subseteq K_{2}^{(+)}$. It follows that $\left|K_{2}^{(+)}: K\right|=\left|\bar{O}_{K}^{0}\right|$, where $K_{2}^{(+)}$is the maximal abelian extension of $K$ unramified at the finite primes such that $\operatorname{Gal}\left(K_{2}^{(+)} / K\right)$ is a direct sum of copies of $\mathbf{Z} / 2 \mathbf{Z}$. Thus by Kummer theory we have that $K_{2}^{(+)}$is the maximal subfield of $K^{(+)}$of the form $K\left(\sqrt{\mu_{1}}, \ldots, \sqrt{\mu_{r}}\right)$ for some $\mu_{i} \in K, i=1,2, \ldots, r$. Similarly, define $K_{2}^{(1)}$ as the maximal abelian extension of $K$ unramified at all primes with $\operatorname{Gal}\left(K_{2}^{(1)} / K\right)$ being a direct sum of copies of $\mathbf{Z} / 2 \mathbf{Z}$. Therefore $K_{2}^{(1)}$ is the maximal subfield of $K^{(1)}$ of the form $K\left(\sqrt{\alpha_{1}}, \ldots, \sqrt{\alpha_{s}}\right)$ for some $\alpha_{i} \in K, i=1,2, \ldots, s$. Furthermore, we note that $K_{2}^{(1)}=K_{2}^{(+)} \cap K^{(1)}$.

Now if $O_{K}^{+}$denotes the subgroup of $O_{K}$ consisting of totally positive integers, then $\mu \in O_{K}^{+} \cap O_{K}^{0}$ with $\mu \notin O_{K}^{2}$ if and only if $K \subsetneq K(\sqrt{\mu}) \subseteq K_{2}^{(1)}$, from which it follows that $\left|K_{2}^{(1)}: K\right|=\left|\bar{O}_{K}^{+}\right|$.

Now we are in a position to prove Theorem 2.5 under the assumption that $K$ is a finite real extension of $Q$ with abelian Galois group $G$.

Proof. Let $M$ be a simple $F_{2} G$-module where $F_{2}$ is the field of two elements. Then by Schur's lemma $M \cong F_{2} G e$ for some idempotent $e$. Now let $\psi$ be the standard involution of $F_{2} G$ given by $\psi(g)=g^{-1}$ for all $g \in G$. Then by the same argument as in the proof of [5, Theorem, p. 615] we have that $F_{2} G e \cong F_{2} G \psi(e)$, resulting from -1 being a power of 2 modulo $n$. Hence we have shown that all simple $F_{2} G$-modules are self-dual. Therefore from [11, Corollary 1, p. 157] we have that $\operatorname{dim}_{2} \bar{U}_{K}^{+} \leqslant \operatorname{dim}_{2} C_{K}$.

By the self-duality established above, we may use exactly the same reasoning as used by Taylor on $\bar{U}_{K}^{+}$and $\bar{U}_{K}^{0}$ in $\left[11,(*)\right.$, p. 157] to establish $\bar{O}_{K}^{+} \cong \bar{O}_{K}^{0}$. Hence by the discussion preceding the proof, we have $K_{2}^{(1)}=K_{2}^{(+)}$; i.e., $\operatorname{dim}_{2} C_{K}=\operatorname{dim}_{2} C_{K}^{(+)}$. Q.E.D.

In what follows, the signature map from $U_{K}$ to $F_{2} G$ is defined by $\operatorname{sgn}(u)=$ $\sum_{\sigma \in G} s\left(u^{\sigma}\right)$, where $s$ is called the signature of $u$ with $s: K^{*} \rightarrow F_{2}$ defined by $s(k)=0$ if $k>0$ and $s(k)=1$ if $k<0$.

It is interesting to note that Lagarias [7] has proved the equivalence of

(2.7) $\operatorname{dim}_{2} C_{K}=\operatorname{dim}_{2} C_{K}^{(+)}$.

(2.8) All odd singular integers $\alpha$ have their signature type determined by the congruence class of $\alpha$ modulo 4 .

(2.9) There are $\alpha$ of all signature types with $\alpha$ an odd singular integer.

(2.10) $K_{2}^{(+)}$is totally real.

Thus, it would be of interest to investigate those totally real algebraic number fields $K$ for which the Sylow 2-subgroup of $\operatorname{Gal}\left(K^{(+)} / K\right)$ is elementary abelian. It is not enough to know that the Sylow 2-subgroup of $\operatorname{Gal}\left(K^{(1)} / K\right)$ is elementary abelian. For example in [2] we see that "most" of the Sylow 2-subgroups of $\operatorname{Gal}\left(K^{(1)} / K\right)$ are elementary abelian where $K$ is a cyclic cubic field. However, there 
are no instances known to the authors in the cyclic cubic case where $\operatorname{Gal}\left(K^{(+)} / K\right)$ has elementary abelian Sylow 2-subgroup.

\section{REFERENCES}

1. C. Chevalley, Deux théorèmes d'arithmétique, J. Math. Soc. Japan 3 (1951), 36-44.

2. V. Ennola and R. Turenen, On cyclic cubic fields, Math. Comp. (to appear).

3. D. Garbanati, Units with norm -1 and signatures of units, J. Reine Angew. Math. 283 / 284 (1976), 164-175.

4. E. Hecke, Lectures on the theory of algebraic numbers, Graduate Texts in Math., no. 77, SpringerVerlag, New York, 1981.

5. I. Hughes and R. Mollin, Totally positive units and squares, Proc. Amer. Math. Soc. 87 (1983), 613-616.

6. G. J. Janusz, Algebraic number fields, Academic Press, New York, 1973.

7. J. C. Lagarias, Signatures of units and congruences $(\bmod 4)$ in certain totally real fields, J. Reine Angew. Math. 320 (1980), 1-5.

8. W. Narkiewicz, Elementary and analytic theory of algebraic numbers, PWN, Warsaw, 1974.

9. B. Oriat, Relation entre les 2-groupes des classes d'ideaux au sens ordinaire et restreint de certain corps de nombres, Bull. Soc. Math. France 104 (1976), 301-307.

10. G. Shimura, On abelian varieties with complex multiplication, Proc. London Math. Soc. (3) 34 (1977), 65-86.

11. M. Taylor, Galois module structure of class groups and units, Mathematika 22 (1975), 156-160.

Department of Mathematics, San Jose State University, San Jose, California 95192 (Current address of H. M. Edgar and B. L. Peterson)

Department of Mathematics, University of Calgary, Calgary, Alberta, T2N 1N4, Canada (Current address of R. A. Mollin) 